\title{
Multiplexer and Variable Optical Attenuator Based on PDLC for Polymer Optical Fiber Networks
}

\author{
P. C. Lallana', C. Vázquez , B. Vinouze ${ }^{2}$, K. Heggarty ${ }^{2}$, \\ and D. S. Montero ${ }^{1}$ \\ ${ }^{1}$ Electronics Technology Department, Carlos III University of Madrid, \\ Leganés, Madrid, Spain \\ ${ }^{2}$ Optics Department, TELECOM Bretagne, Brest, France
}

In this work, a combination of a multiplexer and a variable optical attenuator in the same device (VMUX) for use in Polymer Optical Fiber is proposed. Polymer Dispersed Liquid Crystals (PDLC) are polarization independent, have high contrast and gray scale capability. For these reasons, PDLC cells with pixels can be used as switching elements in the device. Characterization of the PDLC has been carried out at two wavelengths. VMUX Complete switching is reached for driving voltages of $20 \mathrm{Vrms}$, with insertion losses less than $1.6 \mathrm{~dB}$, attenuation larger than $31 \mathrm{~dB}$, rise time less than $2.6 \mathrm{~ms}$ and decay time better than $12.4 \mathrm{~ms}$ have been obtained.

Keywords: coarse wavelength division multiplexing; multiplexer; polymer dispersed liquid crystals; polymer optical fiber; variable optical attenuator

\section{INTRODUCTION}

Polymer Optical Fiber (POF) is being used in a large number of applications: transmission in medical equipment, multimedia applications for civil aviation or in high range cars [1], in home systems [2] or in optical sensor networks [3,4]. POF is lightweight, immune against electromagnetic interferences, is safe for the environment, has no

This work was partially supported by the CICYT (TEC2006 13273 C03 03 MIC), by the European Commission through the NoE EphotonOne+ and BONE (Project no 216863), FACTOTEM CM (S 0505/ESP/000417). Thanks to the Vicerrectorado de Investigación of Carlos III University of Madrid for the prethesis researcher mobility grant. We wish to thank to J. M. S. Pena, J. M. Otón and J. Zubía for comments.

Address correspondence to P. C. Lallana, Electronics Technology Department, Carlos III University of Madrid, C/Butarque 15, Leganés, Madrid 28911, Spain. E mail: pcontrer@ing.uc3m.es 
explosion risk under flammable atmospheres, is easy in handling and connecting, which reduces installation expenses, and it exhibits a large bandwidth for distances less than one hundred meters. In order to increase data transmission capacity, Coarse Wavelength Division Multiplexing (CWDM) can be performed over POF. Two visible wavelength multiplexing has been implemented over 20 meters length POF in [5], and a duplex data and voice signal transmission using CWDM is described in [6]. For longer distances, more than one hundred meters, Perfluorinated Graded Index POF (PF GI-POF) has a low loss wavelength range from 500 to $1300 \mathrm{~nm}[7,8]$, that allows the implementation of CWDM.

Due to the recent development of POF and PF GI-POF networks it could be interesting to develop optical switching elements that work in a broad wavelength range. Many technologies have been reported for optical switching. Micro-electromechanical systems (MEMs) [9,10] that use mobile parts have good fiber to fiber coupling and a great integration scale. On the other hand, switches based on liquid crystal cells [11-15] are used instead others technologies because they do not use mobile parts, they need low excitation voltages and have low consumption. However, most of them have complex structures with a great amount of components [11]. Few components are used in [12] but a fiber optic circulator is needed. A liquid crystal based optical switch capable of operating in a broad wavelength range is given in [13], but only one polarization is obtained at the selected output port, so it has an intrinsic $3 \mathrm{~dB}$ loss. A polarization independent $3 \times 1$ optical multiplexer based on nematic liquid crystals is presented in [14]. Ref. [15] is based on ferroelectric liquid crystal cells that exhibit faster response times but are able to operate in a smaller wavelength range.

In addition, in those networks that involve WDM, it could be necessary to control the optical power level in each wavelength, equalizing them conveniently in order to avoid saturation in those wavelengths whose optical power is high enough. This can be done using a Variable Optical Attenuator (VOA). There are different ways to implement a VOA [16,17]; some of them are based on MEMs technology [18,19]. An interesting VOA with good response times and small size based on transparent electro-optics ceramics is presented in [20]. Different attenuators based on PDLC designed for being used at $1550 \mathrm{~nm}$, are described in [21-23].

As it has been stated before, different systems have been proposed in the literature for implementing optical switches, and some other designs have been presented for developing a VOA. With an insight into the advancement in multifunction integration, development of flexible integrated systems with more functionality and compactness 
into one device might be the potential solution to reduce system cost and complexity. An integration of a Variable Optical Attenuator, multiplexer and switch functions in a single-function device is 70 proposed in [24].

In the present work, a combination of an optical multiplexer and a VOA in the same device (VMUX) is presented. In section 2 an explanation of the proposed VMUX structure, based on PDLC, for being used in POF (even GI-POF) networks is shown. A description of the operation principle is given in section 3. Section 4 includes measurements done for probing that a PDLC sample can be used in the VMUX implementation. Finally, some conclusions are given.

\section{DEVICE STRUCTURE}

The proposed design is a combination of an $\mathrm{N} \times 1$ Multiplexer with a

Variable Optical Attenuator (VOA). An $\mathrm{N} \times 1$ Multiplexer is a system capable of joining $N$ optical inputs in a single output. On the other hand, a Variable Optical Attenuator (VOA) is a device with one input and one output that can modify its transmission reducing the optical power at its output. Then, the VOA-N $\times 1$ Multiplexer (VMUX) is capable of attenuating each input port independently of the others, even switching it off, and finally combining them into a single output optical fiber. A symbol of the VMUX, is shown in Figure 1.

A possible implementation of the VMUX is using the $3 \times 1$ optical multiplexer given in [14]. The structure is based on nematic liquid crystal cells, polarizing beam splitters and lenses. It is possible achieving variable attenuation for each input by modifying the voltage applied to the liquid crystal pixels. A simpler implementation, using fewer components, it is proposed in Figure 2. It is composed of a Polymer Dispersed Liquid Crystal cell with as many pixels as input

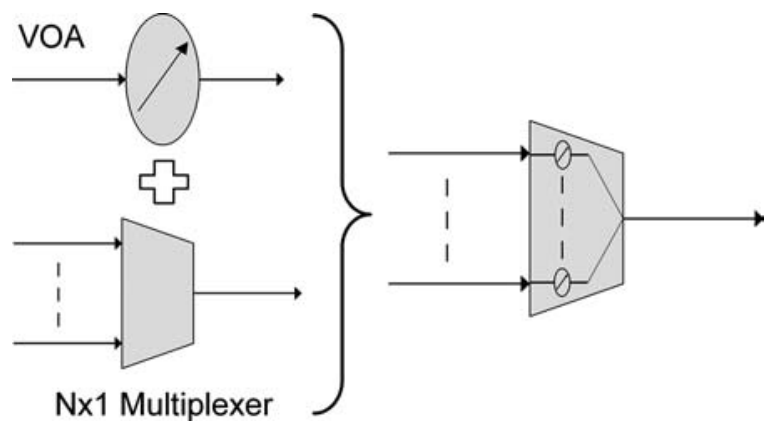

FIGURE 1 Symbol of the VMUX. 


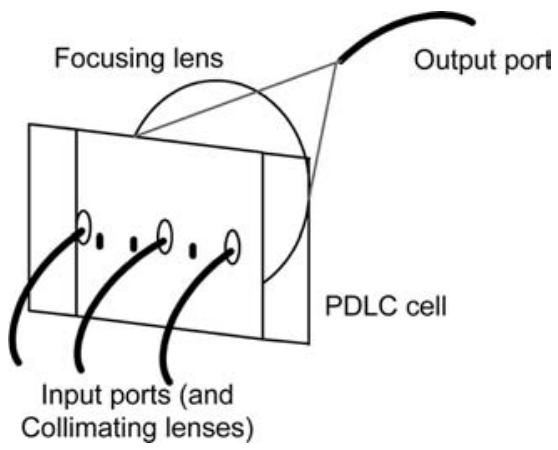

FIGURE 2 Schematic of the proposed VMUX.

ports, each pixel controls light coming from each input port, finally a single lens is used for focusing light from all the inputs in the output port. Each input port needs a lens for collimating light that comes from each optical fiber.

PDLC are polarization independent, they do not need polarizers neither polarizing beam splitting for switching, like occurs with pure nematic liquid crystals. For this reason fewer elements are needed and less insertion losses are expected. Furthermore, it is supposed that the Polarization Dependent Losses (PDL) will be also smaller.

Proposed VMUX gives more flexibility than common multiplexer. This is because common multiplexer is only able to perform Wavelength Division Multiplexing (WDM) over a fiber, their input ports work for a single wavelength. Ports of the VMUX are wavelength independent; they work in the same way for all the wavelengths and independently of the other input ports. For this reason apart of WDM, Time Division Multiplexing can also be implemented, even a mixture of TDM and WDM, i.e., WDM performed in different fibers can be temporally multiplexed into a single fiber. Furthermore, variable attenuation can be applied to each input port. In this way, equalization of the different wavelengths can be implemented, for example in optical fiber amplifier, if this device will be used in other type of networks.

Different setups of the VMUX are possible because the proposed schema manages light from each input port independently of the others. Then, there are the following functions at the system output:

- There is no light from any port.

- Only light from one port reach to the output.

- A combination of light from two or more inputs gets to the output.

- Light of all inputs passes through the device. 
In addition to the functions explained before, the VMUX is capable of 125 adding an extra attenuation to each input port that passes through it, giving more flexibility to the design.

\section{DEVICE OPERATION PRINCIPLE}

In this section a brief description of the $\mathrm{N} \times 1$ Multiplexer combined with a Variable Optical Attenuator (VMUX) implemented with Polymer Dispersed Liquid Crystal (PDLC) is given. A PDLC cell with as many pixels as the multiplexer input ports can be used as the switching element in the VMUX.

PDLC consist of a matrix of polymer material where microdroplets with molecules of liquid crystals are dispersed. This mixture is sandwiched between glasses covered with ITO, a transparent conductor, which allows applying to the mixture an electric field. Structure of the PDLC is shown in Figure 3. When no voltage is applied to the PDLC cell, the liquid crystal molecules that form the microdroplets have not preferential orientation. In this scenario, the structure presents an inhomogeneous refraction index that causes the incoming light to be strongly scattered. The output beams are not parallel to the incoming light, the diffusing state can be seen in Figure 3(a). When an AC electric field is applied to the electrodes that cover the PDLC cell, liquid crystals molecules are reoriented in the field direction. In this situation, the polymer matrix and the microdroplets exhibit almost the same refraction index in the light propagation direction, because the polymer matrix is designed to have the ordinary refraction index

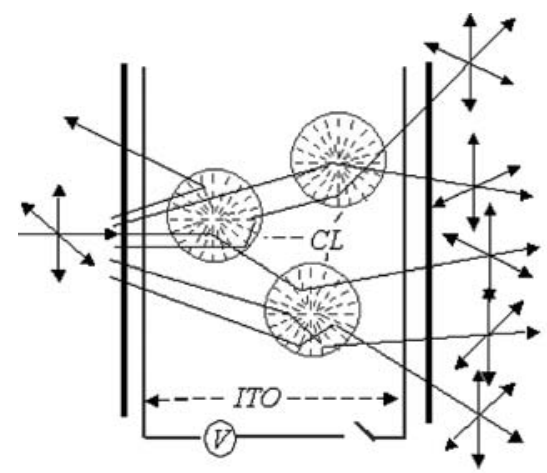

(a)

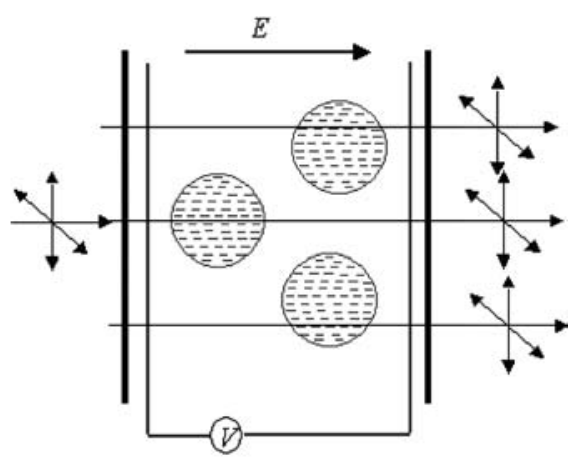

(b)

FIGURE 3 Internal structure of a not excited PDLC (a) and when an electric field is applied (b). 
of the liquid crystal that forms microdroplets. This causes the optical beams passing through the device parallel to the incoming light, the transmitting state is shown in Figure 3(b). Gray scales are achieved because liquid crystal molecules orientation depends on the voltage applied to the PDLC cell. The size of the microdroplets of liquid crystal and their density in the polymer matrix determine device transmission and its response characteristics.

In summary, the PDLC can acts as optical switch, light can pass through the device when an AC voltage is applied to the cell, and incoming light is not able to pass through the PDLC when no voltage is applied. Intermediate voltages can allow its behavior as a VOA. Furthermore, a PDLC with as many pixels as multiplexer inputs can be used as the switching element of the VMUX.

\section{MEASUREMENTS}

A PDLC cell without pixels has been characterized in order to determine their capability for being used as VMUX. PDLC system used in the set up is a mixture of $80 \%$ by weight of Merck TL205 liquid crystal with $20 \%$ of PN393 monomer. Switching voltage, insertion losses, isolation and response times were measured for several positions of the optical sources at two different wavelengths: $530 \mathrm{~nm}$ and $650 \mathrm{~nm}$. Tests can be split in two types: static and dynamic measurements. The first one corresponds to the PDLC transmission characterization when its excitation is maintained during a long period of time. The second group of measurements evaluate PDLC response times when it switched.

\subsection{Set up}

A diagram of the set up used for characterizing the PDLC is shown in Figure 4. The VMUX is formed by the PDLC sample, the $40 \mathrm{~mm}$ diameter focusing lens and the output fiber ( $200 \mathrm{~cm}$ length POF). PDLC transmission has been measured for several positions of the optical sources respect to the center of the focusing lens, " $r$ ", in order to check the PDLC capability for being used as VMUX. For all the situations the optical sources have been placed close to the PDLC sample, $d<1 \mathrm{~mm}$.

Two visible laser diodes: one at $532 \mathrm{~nm}$ and $0.99 \mathrm{~mW}$ from Hero and the other at $650 \mathrm{~nm}$ and $5 \mathrm{~mW}$ from Power Technology Inc have been used in the test. An iris diaphragm limits the laser beam spot. A $200 \mathrm{~cm}$ length POF joins the output port to the power meter. A Hewlett Packard wave generator has been employed for generating PDLC excitation. 


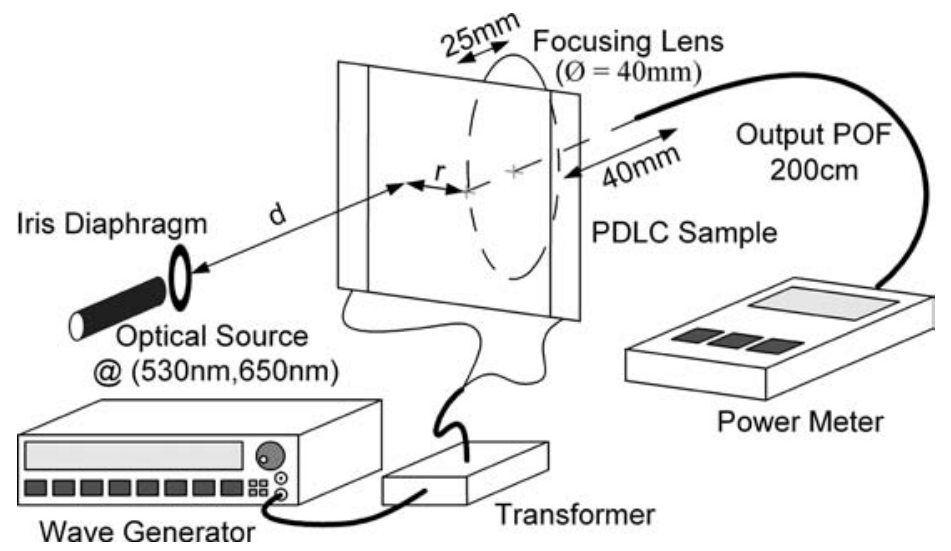

FIGURE 4 Experimental set up for measuring PDLC characteristics.

\subsection{Static Measurements}

This section refers to the measurements done when the excitation of the liquid crystal cells is maintained during a long period of time. PDLC transmission was measured for different amplitudes of a $50 \mathrm{~Hz}$ sinusoidal wave. A 2.4:1 transformer has been utilized for adapting voltage level drove to the PDLC sample. In this way, the switching voltage, insertion losses and attenuation could be determined.

PDLC response at both wavelengths, when the optical source was placed in front of the center of the focusing lens, $r=0$, and when the

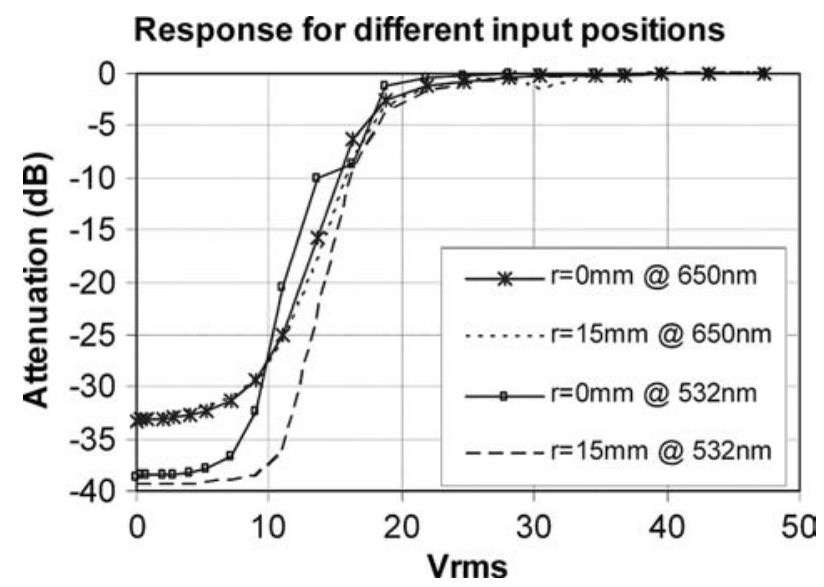

FIGURE 5 PDLC response for different input port positions. (See COLOR PLATE XI) 
optical source was placed in one side, $r=15 \mathrm{~mm}$ from the center of the lens, is shown in Figure 5.

Complete switching is obtained for drive voltages of $20 \mathrm{Vrms}$ in all the situations. Insertion losses are less than $1.6 \mathrm{~dB}$ at $532 \mathrm{~nm}$ and $1 \mathrm{~dB}$ at $650 \mathrm{~nm}$. Attenuation, relationship between the situations when transmission is high and low, is near $39.5 \mathrm{~dB}$ at $532 \mathrm{~nm}$ and $31 \mathrm{~dB}$ at $650 \mathrm{~nm}$. Gray scales are achieved approximately from $0 \mathrm{Vrms}$ to $30 \mathrm{Vrms}$, transmission can be configured modifying the voltage applied to the PDLC. Despite that there are no attenuation differences versus " $r$ " when $650 \mathrm{~nm}$ laser is used, PDLC responses are a bit different at $532 \mathrm{~nm}$.

\subsection{Dynamic Measurements}

This section refers to measurements when the PDLC is switching on/off repeatedly. A digital Tektronix oscilloscope and a SFH $350 \mathrm{~V}$ 210 photodiode with a resistor were used to determine PDLC response times. Two sets of measurements were done. In the first one, a 2.4:1

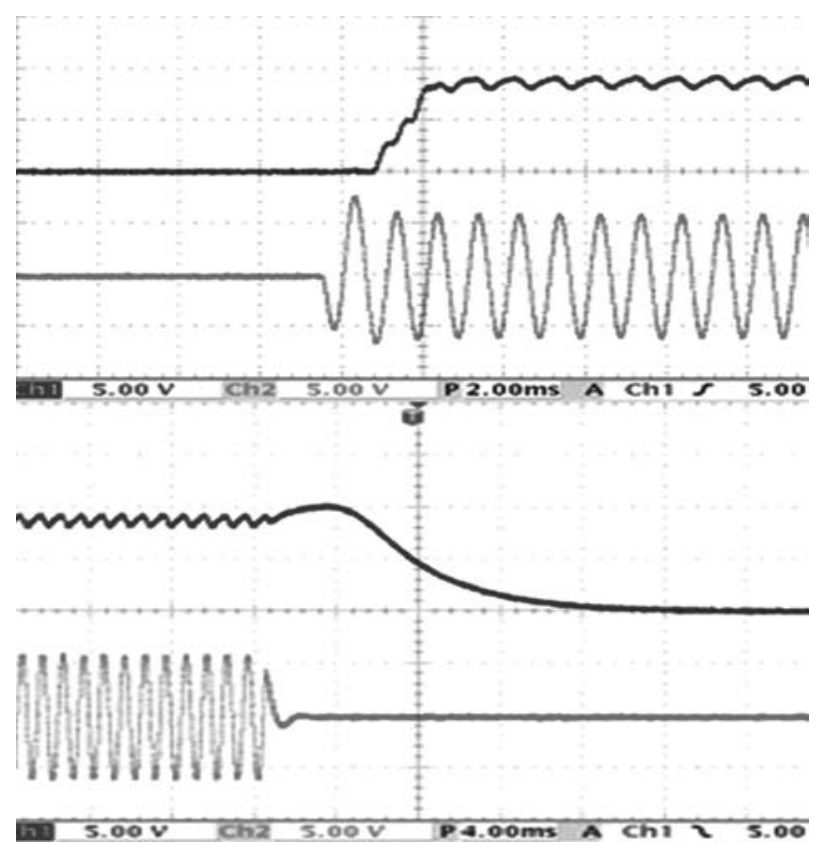

FIGURE 6 PDLC response times at $532 \mathrm{~nm}$ when $1 \mathrm{kHz}$ sinusoidal wave is applied. 


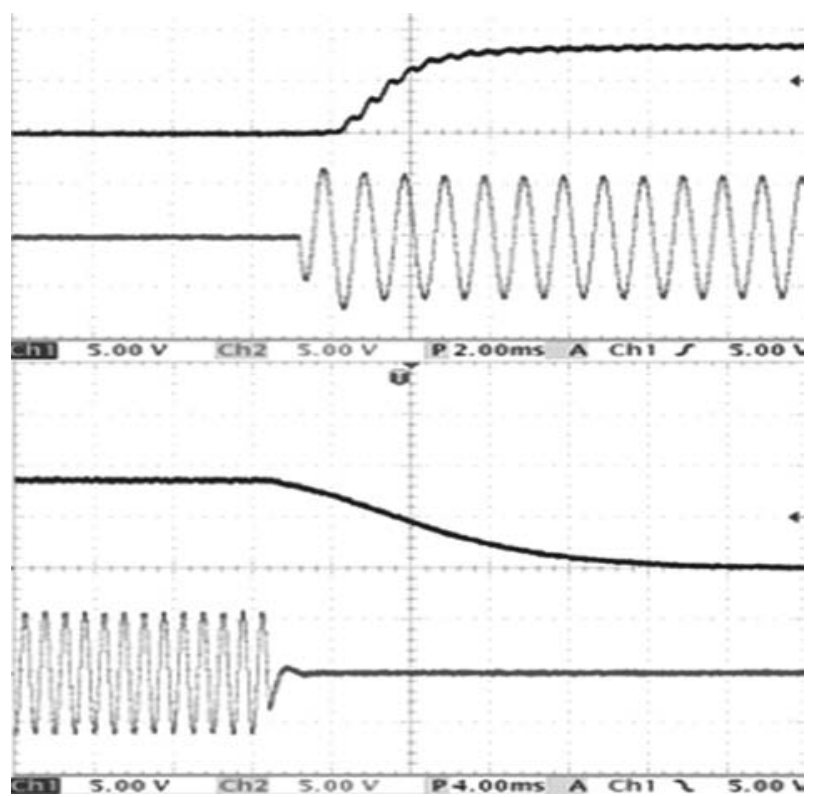

FIGURE 7 PDLC response times at $650 \mathrm{~nm}$ when $1 \mathrm{kHz}$ sinusoidal wave is applied.

transformer was used to drive voltage to the PDLC, whereas in the second group of tests, $a \times 10$ wide bandwidth amplifier from FLC Electronics was utilized.

Figures 6 and 7 show PDLC response times for the first set of measurements. In this case, due to the transformer limitations, a $1 \mathrm{kHz}$ sinusoidal wave is applied to the cell. A $40 \mathrm{Vrms}, 1 \mathrm{kHz}$ sinusoidal wave is applied for $0.5 \mathrm{~s}$ and $0 \mathrm{~V}$ is drove to the PDLC during $0.5 \mathrm{~s}$, in other words, an envelope of $1 \mathrm{~Hz}$ is used for modulating the $40 \mathrm{Vrms}, 1 \mathrm{kHz}$ sinusoidal wave. In both figures, a 10 factor has to be applied to the measurements done with channel 2 (PDLC driving voltage) due to the probe is in $\times 10$ position. Figure 6 describe PDLC response at $532 \mathrm{nmm}$, rise time can be observed in the upper part of the figure and decay time can be seen in the lower section. Excitation signal is depicted below the obtained transmission response. Figure 7 represents the same situation when the $650 \mathrm{~nm}$ optical source was used.

Figures 8 and 9 show PDLC response times for the second set of measurements, at $532 \mathrm{~nm}$ and $650 \mathrm{~nm}$ respectively, where $\mathrm{a} \times 10$ wide bandwidth amplifier was used. In this situation, a $1 \mathrm{kHz}$ square wave 


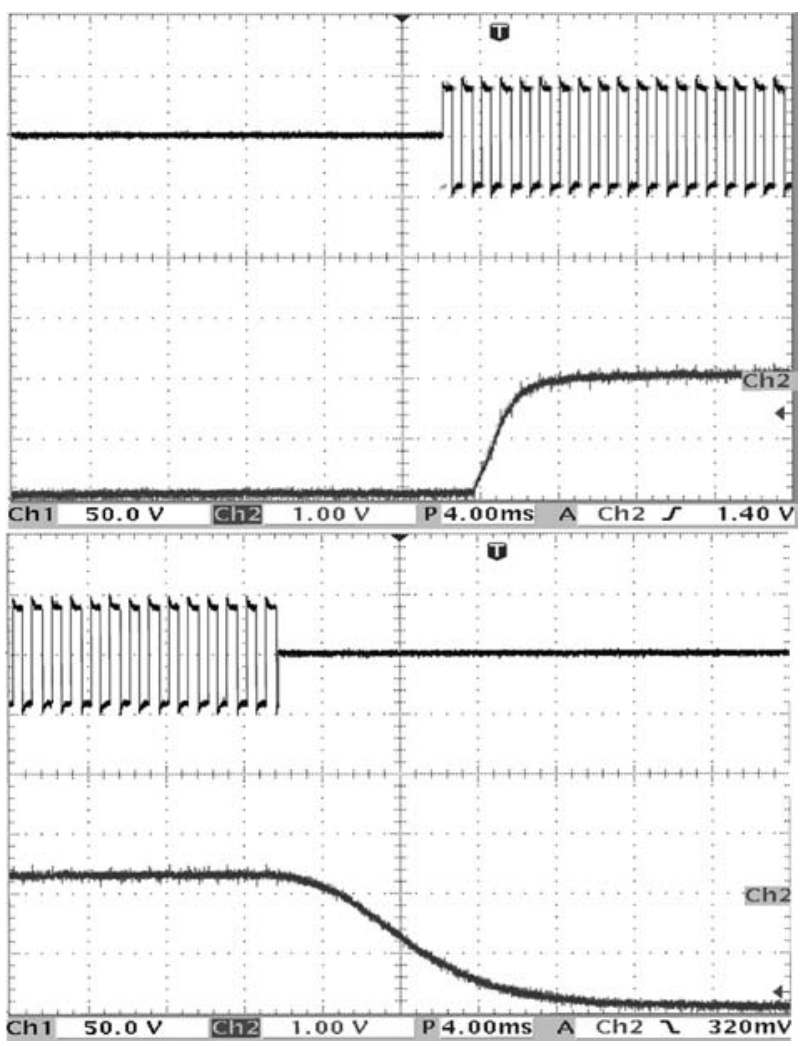

FIGURE 8 PDLC response times at $532 \mathrm{~nm}$ when $1 \mathrm{kHz}$ square wave is applied.

is applied to the sample in the same conditions than the previous ones. These figures maintain similar layout than the previous ones, upper part of each illustration represent rise time at the corresponding wavelength, whereas lower section represents decay time. In this case, 235 the excitation signal is shown over the PDLC response.

It can be seen that similar results are obtained for both wavelengths in the two set of measurements presented. Rise time less than $2.6 \mathrm{~ms}$ and decay time better than $12.4 \mathrm{~ms}$ were obtained. Difference between rise and decay times is in concordance with nematic liquid crystal properties where it is known that relaxation times are larger than excitation times.

A ripple in the PDLC transmission can be observed mainly when a $1 \mathrm{kHz}$ sinusoidal is applied. It seems that the PDLC is able to follow 


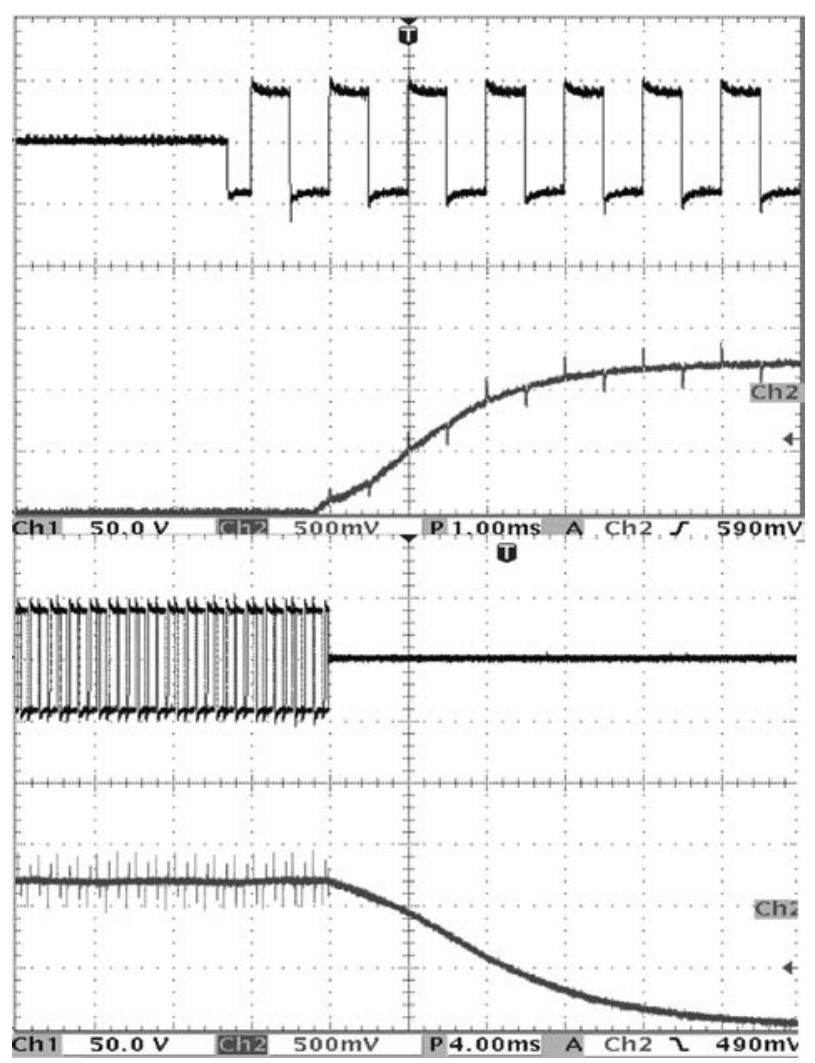

FIGURE 9 PDLC response times at $650 \mathrm{~nm}$ when $1 \mathrm{kHz}$ square wave is applied.

excitation. Ripple is smaller for square wave voltages; it can be 245 suppressed by using a higher frequency (typically $100 \mathrm{kHz}$ ).

\section{CONCLUSIONS}

An $\mathrm{N} \times 1$ optical multiplexer combined with a variable optical attenuator (VMUX) has been proposed. A Polymer Dispersed Liquid Crystal (PDLC) cell with the desired number of pixels can be used for implementing a VMUX with as many inputs ports as cell pixels. Light that comes from each input port is treated by a PDLC pixel. The design is compact, it is made of few components, scalable, more channels can be added using a PDLC cell with more pixels, capable of working simultaneously in a broad wavelength range. In addition, each input port can be switched off or attenuated independently of the others. 
Characterization of a PDLC cell transmission and it response times have been done. Insertion losses less than $1.6 \mathrm{~dB}$ at $530 \mathrm{~nm}$ and better than $1 \mathrm{~dB}$ at $650 \mathrm{~nm}$ have been obtained. Complete switching has been reached for drive voltages of $20 \mathrm{Vrms}$ at both wavelengths. Attenuation near $39.5 \mathrm{~dB}$ at $530 \mathrm{~nm}$ and $31 \mathrm{~dB}$ at $650 \mathrm{~nm}$ has been achieved. Rise time less than $2.6 \mathrm{~ms}$ and decay time better than $12.4 \mathrm{~ms}$ for both wavelengths have been measured. Finally, continuous variable attenuation can be carried out modifying the voltage applied to the PDLC.

\section{REFERENCES}

[1] Kibler, T., Poerl, S., Böck, G., Huber, H. P., \& Zeeb, E. (2004). J. Ligthwave Technol., 22(9), 21842199.

[2] Koonen, A. M. J., van den Boom, H. P. A., Ng'oma, A., Garcia Larrode, M., Zeng, J., \& Khoe, G. D. (Sept. 2005). "POF Application in Home Systems and Local Systems", Proc. POF 2005, Hong Kong, 165168.

[3] Knowles, S. F., Jones, B. E., Purdy, S., \& France, C. M. (1998). Sens. Actuators, A68, 320323.

[4] Vázquez, C., Gonzalo, A. B., Vargas, S., \& Montalvo, J. (2004). Sens. Actuators, A116, 2232.

[5] Yonemura, M., Kawasaki, A., Kato, S., Kagami, M., \& Inui, Y. (2005). Optics Lett., $30(17), 22062208$.

[6] Zhang, Y., Ma, H., Zhang, T., Wang, Z., Wang, D., Zheng, R., Yang, H., \& Ming, H. (2005). Study of coarse wavelength division multiplexing using polymer optical fiber transmission window. Proceedings of SPIE, 5644, 835839.

7] Goudeau, J., Widawski, G., \& Bareel, B., et al. (2004). GI POF for GB Ethernet links. 13th International Plastic Optical Fibres Conf. 76.

[8] Plastic Optical Fiber Trade Organization. (2004). Present State of the Art of Plastic Optical Fiber (POF) Components and Systems, Brighton, MA, USA.

[9] Marxer, C. \& De Rooij, N. F. (1999). J. Lightwave Technol., 17(1), 26.

[10] Jensen, R. A. (2002). Comparing of optical switching technologies for intelligent optical networks, LEOS '02. 1, 230231

[11] Soref, R. A. (1981). Optics Lett., 6(6), 275277.

[12] Sumriddetchkajorn, S., Riza, N., \& Sengupta, D. (2001). Opt. Engin., 40, 15211528.

[13] Vázquez, C., Pena, J. M. S., \& Aranda, A. L. (2003). Optics Communication, 224, 5762.

[14] Lallana, P. C., Vázquez, C., Pena, J. M. S., \& Vergaz, R. (2006). Opto Electronics review, 14(4), 311318.

[15] Riza, A. \& Yuan, S. (1999). J. Light. Tech. Lett., 17, 15751584.

[16] Losert, W., Bauer, G., Ziemann, O., \& H. (2004). Poisel POF variable optical attenuators status and new concepts. 13th International Plastic Optical Fibres

Q3 Conference, Nürnberg, 546551.

[17] Adamopoulos, S., Konstantinou, S., \& Kalymnios, D. (2004). Variable POF attenua tors. 13th International Plastic Optical Fibres Conference, Nürnberg, 567573.

[18] Syms, R. R. A., Zou, H., Stagg, J., \& Veladi, H. (2004). Journal of Micromech. Microeng., 14, 17001710.

[19] Lee, C. \& Lin, Y. S. (2004). IEEE Sensors Journal, 4(4), 503509. 
[20] Jiang, H., Zou, Y. K., Chen, Q., Li, K. K., Zhang, R., \& Wang, Y. Transparent Electro Optics Ceramics and Devices, Boston Applied Technologies, Inc.: Woburn, 305 MA, USA, 01801.

[21] Hirabayashi, K., Wada, M., \& Amano, C. (2001). Applied Optics, 40(21), 35093517.

[22] Ramanitra, H., Chanclou, P., Vinouze, B., \& Dupont, L. (2003). Mol. Cryst. Liq. Cryst., 404, 5573.

[23] Chanclou, P., Vinouze, B., Roy, M., \& Cornu, C. (2005). Optics Communication, 248, 310 167172.

[24] Lallana, P.C., Vàzquez, C., Montero, D. S., Heggarty, K., \& Vinouze, B. (2007). Dual $3 \times 1$ multiplexer for POF networks, 17th International Conference on Plastic Optical Fibers ICPOF '07. 